\title{
MicroRNAs in Celiac Disease Diagnosis: A miR Curiosity or Game-Changer?
}

\author{
Paolo Giuffrida $^{1}$ - Antonio Di Sabatino ${ }^{1}$
}

Published online: 22 January 2020

(c) Springer Science+Business Media, LLC, part of Springer Nature 2020

Celiac disease (CD), an immune-mediated enteropathy triggered by gluten intake in genetically predisposed patients, is globally the most prevalent known enteropathy [1]. In adults, $\mathrm{CD}$ diagnosis is based on the positivity of serum antiendomysial IgA antibodies and/or anti-tissue-transglutaminase IgA antibodies, confirmed by typical histopathological duodenal lesions such as villous atrophy, increased intraepithelial lymphocytes, and crypt hyperplasia [1,2]. The only approved treatment for $\mathrm{CD}$ is adherence to a strict glutenfree diet (GFD), although several drugs have been investigated in clinical trials. In addition to serum anti-endomysial $\operatorname{Ig} \mathrm{A}$ and anti-tissue-transglutaminase $\operatorname{Ig} \mathrm{A}$ antibodies [2], several biomarkers, including plasma citrulline, serum intestinal fatty acid-binding protein, and serum regenerating islet-derived 3- $\alpha$, have been considered for $\mathrm{CD}$ diagnosis [3-5], with the proviso that all had good accuracy only in the presence of a concomitant small bowel villous atrophy, i.e., in $\mathrm{CD}$ patients consuming gluten in the diet, similar to the aforementioned antibodies [3-5].

MicroRNAs (miRs) are single-stranded noncoding RNAs of 21-23 nucleotides able to inhibit the translation of multiple mRNAs, thus silencing their targeted genes. Over the last few years, miRs have gained attention in many disorders due to their clinical applications in diagnosis and therapy [6]. Several miRs have been evaluated as potential biomarkers in a number of gastrointestinal disorders, including $C D$, inflammatory bowel disease, and colorectal and gastric cancer. In particular, many studies investigated the presence of miRs in the small bowel mucosa of CD patients, either at diagnosis or after consuming a GFD in order to clarify their pathological mechanisms in this condition [7]. To our knowledge, circulating miRs have been assessed

Paolo Giuffrida

paolo.giuffrida01@universitadipavia.it

1 First Department of Internal Medicine, San Matteo Hospital Foundation, University of Pavia, Piazzale Golgi 19, 27100 Pavia, Italy so far only in pediatric CD patients $[8,9]$. Buoli Comani et al. [8] reported a lower expression of plasma miR-192-5p and miR-31-5p in pediatric CD patients at diagnosis than in controls. Conversely, plasma levels of miR-21-5p were higher in pediatric CD patients with severe villous atrophy at diagnosis than in controls. Expression of miR-192-5p, miR-31-5p, and miR-21-5p in the plasma of pediatric CD patients at diagnosis and controls was consistent with duodenal histopathology. Plasma miR-192-5p was also decreased in pediatric $\mathrm{CD}$ patients consuming a GFD in comparison with controls, whereas plasma miR-31-5p and miR-21-5p returned to normal levels after at least 1 year of a GFD. Amr et al. [9] demonstrated an overexpression of miR-21 and a down-regulation of miR-31 in the plasma of untreated pediatric CD patients in comparison with healthy controls, without any significant difference between treated pediatric $\mathrm{CD}$ patients and controls.

In this issue of Digestive Diseases and Sciences, Bascuñán et al. [10] reported a two-center Chilean/Italian study of 30 adult CD patients, 10 at CD diagnosis and 20 on a GFD for at least 1 year. In this study [10], the authors evaluated the pro-inflammatory miR-146a, miR-155, miR-21, and miR-125b in peripheral blood mononuclear cells (PBMCs), monocytes, intestinal mucosa, and plasma of these patients. Levels of miR-146a, miR-155, and miR-21 in PBMCs, miR155 in monocytes, and miR-155, miR-21, and miR-125b in plasma were elevated in both untreated and treated CD patients. In vitro exposure with gliadin markedly up-regulated miR-155 expression in CD PBMCs and monocytes, whereas miR-155 and miR-21 were increased in CD monocytes stimulated in vitro with both gliadin and interferon- $\gamma$ in comparison with baseline levels. The expression of miR in the intestinal mucosa did not change among all groups. MiR-146a and miR-155 expression showed high sensitivity and specificity for $\mathrm{CD}$, irrespective of the current dietary treatment.

Altogether, these results highlight that inflammationrelated miRs are elevated in the peripheral blood of patients 
with $\mathrm{CD}$, and selected miRs might be used as potential diagnostic biomarkers for CD. Nevertheless, there are several limitations of this study: Firstly, no differences of miR-146a, miR-155, miR-21, and miR-125b in PBMCs, monocytes, and plasma were identified between untreated and treated CD patients. These miRs appeared to be related to the $\mathrm{CD}$ itself, regardless of treatment and subsequent mucosal healing. Nonetheless, the authors' findings with only $30 \mathrm{CD}$ patients need to be confirmed and validated in much larger cohorts before drawing final conclusions that are aimed at changing the daily clinical practice in CD. Furthermore, assessing inflammation-related miRs along the broad clinical spectrum of $\mathrm{CD}$, from latency to refractory stage, would be useful. Secondly, miR-146a, miR-155, miR-21, and mi-RNA-125b were increased in other autoimmune and/or inflammatory disorders, such as inflammatory bowel disease, rheumatoid arthritis, systemic lupus erythematosus, multiple sclerosis, and Alzheimer's disease [10]. Thirdly, it is surprising that in this study [10] no differences of genotyping were identified between $\mathrm{CD}$ and healthy groups in terms of human leukocyte antigen (HLA)-DQ2, HLA-DQ8 and HLA-DQ7 haplotypes, known to be present in all CD patients and in $30 \%$ of the general population. As HLA-DQ2/DQ8/DQ7 are the primary genetic susceptibility loci in $\mathrm{CD}$, it is desirable to have HLA-DQ2, HLA-DQ8, and HLA-DQ7 haplotypes in 30\% of controls in studies investigating differential miR expression in $\mathrm{CD}$ in order to differentiate the genetic backgrounds between the $\mathrm{CD}$ and control groups.

The novelty and potential power of miR-based diagnosis has fueled considerable investment in this technology by the biotechnology industry, built in some instances on inflated expectations [6]. Notwithstanding, the preliminary reports on circulating miRs as biomarkers in $\mathrm{CD}$ [8-10] need to be weighed with caution until confirmatory studies are conducted, since research on miRs as diagnostic biomarkers in CD is in its infancy. The major strength of the current study [10] is the assessment for the first time in adults affected by $\mathrm{CD}$ of circulating miRs, not only in plasma, but also in PBMCs and peripheral monocytes. In keeping with the results derived from pediatric $\mathrm{CD}$ cohorts [9], Bascuñan et al. [10] confirmed an increase in plasma miR-21 in adults with untreated $\mathrm{CD}$. Conversely, unlike previous results in CD children on a GFD [9], plasma miR-21 was increased in treated CD adults [10]. Thus, further investigations are needed to clarify whether these findings regarding miR-21 in treated CD are secondary to the different ages of the patients. Furthermore, all of the three studies on circulating miRs in CD [8-10] recruited small numbers of patients, namely 17,25 , and 10 with untreated CD and 7, 25, and 20 with treated CD, respectively. Surely, these doubts would be best addressed with future studies on circulating miRs in larger cohorts of $\mathrm{CD}$ patients. Considering the aforementioned issues, the possible uses of miRs as noninvasive biomarkers in CD need extensive confirmation prior to clinical application. As circulating levels of several miRs, including miR192-5p, miR-146a, miR-155, miR-21, and miR-125b, do not differ between untreated and treated $\mathrm{CD}$, they might be additional diagnostic tools in patients without a final diagnosis of $\mathrm{CD}$, but already on a GFD. On the other hand, microRNAs altered only in patients with untreated $\mathrm{CD}$, but not in those with treated $\mathrm{CD}$, might be considered as predictors of small villous atrophy and also as possible noninvasive markers of treatment efficacy. These would be of great interest in order to follow-up patients with potential CD and to monitor adherence to GFD in treated $\mathrm{CD}$, respectively. As the first assessment of circulating miRs as potential biomarkers in adults with $C D$, this study has considerable potential pending larger and more comprehensive investigation of this promising class of biomarkers.

Author's contribution PG and ADS participated in the drafting of the manuscript or critical revision of the manuscript for important intellectual content and provided approval of the final submitted version.

\section{Compliance with Ethical Standards}

Conflict of interest The authors declare that they have no conflict of interest.

\section{References}

1. Di Sabatino A, Lenti MV, Giuffrida P, Vanoli A, Corazza GR. New insights into immune mechanisms underlying autoimmune diseases of the gastrointestinal tract. Autoimmun Rev. 2015;14:1161-1169.

2. Di Sabatino A, Biagi F, Lenzi M, et al. Clinical usefulness of serum antibodies as biomarkers of gastrointestinal and liver diseases. Dig Liver Dis. 2017;49:947-956.

3. Fragkos KC, Forbes A. Citrulline as a marker of intestinal function and absorption in clinical settings: A systematic review and meta-analysis. United Eur Gastroenterol J. 2018;6:181-191.

4. Adriaanse MP, Tack GJ, Passos VL, et al. Serum I-FABP as marker for enterocyte damage in coeliac disease and its relation to villous atrophy and circulating autoantibodies. Aliment Pharmacol Ther. 2013;37:482-490.

5. Marafini I, Di Sabatino A, Zorzi F, et al. Serum regenerating isletderived 3-alpha is a biomarker of mucosal enteropathies. Aliment Pharmacol Ther. 2014;40:974-981.

6. Bonneau E, Neveu B, Kostantin E, Tsongalis GJ, De Guire V. How close are miRs from clinical practice? A perspective on the diagnostic and therapeutic market. EJIFCC. 2019;30:114-127.

7. Felli C, Baldassarre A, Masotti A. Intestinal and circulating MicroRNAs in coeliac disease. Int J Mol Sci. 2017;18:1907.

8. Buoli Comani G, Panceri R, Dinelli M, et al. miR-regulated gene expression differs in celiac disease patients according to the age of presentation. Genes Nutr. 2015;10:482.

9. Amr KS, Bayoumi FS, Eissa E, Abu-Zekry M. Circulating microRNAs as potential non-invasive biomarkers in pediatric 
patients with celiac disease. Eur Ann Allergy Clin Immunol. 2019;51:159-164.

10. Bascuñán KA, Pérez-Bravo F, Gaudioso G, et al. A miR-based blood and mucosal approach for detecting and monitoring celiac disease. Dig Dis Sci. (Epub ahead of print). https://doi. org/10.1007/s10620-019-05966-z.
Publisher's Note Springer Nature remains neutral with regard to jurisdictional claims in published maps and institutional affiliations. 